\section{Meta-análise de ensaios clínicos de intervenção \\ familiar na condição esquizofrenia}

\author{
Meta-analysis of clinical trials on family \\ intervention in schizophrenia
}

\section{Abstract}

The present study aimed to assess the efficacy of cognitive-behavioral family interventions by relatives of schizophrenic patients under community care, specifically targeting relapse and family burden as outcomes. Independent researchers conducted the analyses of the pertinence and quality of trials identified through a search strategy, following a previously developed protocol. Eleven randomized or quasi-randomized trials were selected. The summary relative risk of relapse using the fixed effects model was favorable to family intervention, with estimated efficacy reaching nearly 60\% (50\%-70\%). Summary relative risk in the cognitive-behavioral therapy trials subgroup $[R R=0.43(0.28-0.67)]$ was equivalent to that of the behavioral therapy subgroup $[R R=$ 0.37 (0.23-0.60)] and the "pragmatic" subgroup $[R R=0.37(0.21-0.66)]$, although the "pragmatic" trials were generally analyzed for effective treatment. The difference in summary overall risk of relapse was nearly 30\% using the random effects model. Only four trials analyzed family burden as outcome, including different dimensions of burden. Results of individual trials were generally favorable to family intervention, for both the objective and subjective dimensions.

Schizophrenia; Randomized Controlled Trials; Meta-Anlaysis
Maria Goretti Andrade Rodrigues 1

Letícia Krauss-Silva 1

Ana Cristina Marques Martins 2

\section{Introdução e justificativa}

A esquizofrenia é um dos mais graves transtornos mentais e atinge aproximadamente $1 \%$ da população mundial. Caracteriza-se por uma desorganização de diversos processos mentais e por alteração do comportamento, evoluindo com recaídas (recorrência de sintomas), na maioria dos casos, que podem resultar em que a vida afetiva, social e produtiva do paciente fique severamente comprometida. Pode representar ainda um impacto importante na vida dos familiares, o que vem sendo conceituado como "sobrecarga familiar", bem como custos relevantes para toda a sociedade. No Brasil, a esquizofrenia corresponde ao $5^{\circ}$ lugar na manutenção de auxílio-doença no país 1 .

Para o presente trabalho, os procedimentos de atenção à condição esquizofrenia, inclusive a intervenção familiar, foram identificados, inicialmente, a partir do livro-texto de Kaplan \& Sadock 2, e de documentos governamentais do Canadá ${ }^{3}$ e dos Estados Unidos ${ }^{4}$. Esse último documento aponta que das intervenções psicossociais testadas para o tratamento da esquizofrenia, a partir da década de 70, apenas para a chamada "intervenção familiar", haveria um volume de ensaios clínicos bem desenhados com resultados consistentes

A análise das propostas de intervenção familiar dos ensaios foi previamente efetuada $5 \mathrm{com}$ 
base em teorias, métodos e técnicas comportamentais e cognitivo-comportamentais 6,7,8,9.

A partir daqueles referenciais, foi então considerado "comportamental" o ensaio de intervenção familiar que apresentasse técnicas baseadas na teoria do condicionamento clássico, na teoria do condicionamento operante ou na teoria da aprendizagem social, privilegiando-se dentro desse último grupo, os procedimentos do treinamento de habilidades sociais. Consideramos, então, "cognitivo-comportamental" o ensaio cuja abordagem na intervenção familiar: (1) apresentasse as seguintes proposições fundamentais 9 (a) a atividade cognitiva afeta o comportamento (modelo mediacional), (b) a atividade cognitiva pode ser monitorada e alterada, e (c) a mudança do comportamento desejada pode ser alcançada através da mudança cognitiva; (2) aplicasse as terapias baseadas no método cognitivo-comportamental - terapias de reestruturação cognitiva, terapias de habilidades para lidar com dificuldades e terapias de resolução de problemas. Beck 7 esclarece que o ponto focal dessas terapias difere, sobretudo, porque o terapeuta cognitivo-comportamental direciona sua técnica para modificar o conteúdo ideacional envolvido no sintoma, através da mediação cognitiva.

O grupo de ensaios denominados "pragmáticos" apresenta características comuns quanto aos objetivos e semelhanças de conteúdos/extensão da intervenção proposta, e quanto ao fato de não constituírem uma técnica, derivada de base teórico-metodológica definida, mas, de constituírem fragmentos de técnicas comportamentais e cognitivo-comportamentais, ou seja, pedaços de procedimentos utilizados sem a lógica e a articulação previstas.

As revisões sistemáticas analisam estudos com base em protocolo previamente desenhado e contribuem para integrar, de forma adequada, as informações desses estudos, fornecendo uma base científica para a tomada de decisão racional no setor saúde 10,11. A meta-análise é um procedimento estatístico que pode ser utilizado nas revisões sistemáticas para sintetizar aspectos quantitativos de estudos, fornecendo medidas sumárias para os desfechos estudados.

A presente revisão pretendeu contribuir para a avaliação da conveniência da incorporação de procedimentos, de base comportamental e cognitivo-comportamental, dirigidos a familiares de esquizofrênicos, como componente relevante da atenção extra-hospitalar, comunitária, a esse grave problema de saúde mental. As revisões sistemáticas existentes sobre a eficácia da intervenção familiar na condição esquizofrenia $12,13,14,15,16$ não preencheram o objetivo do presente estudo, além de apresentarem, em geral, li- mitações metodológicas importantes, conforme referido adiante.

\section{Objetivo}

O objetivo da revisão sistemática/meta-análise foi avaliar a eficácia da intervenção familiar baseada no método comportamental, no método cognitivo-comportamental ou em procedimentos "pragmáticos" na condição esquizofrenia, com relação aos desfechos recaída e sobrecarga familiar.

\section{Metodologia}

\section{Identificação de revisões sistemáticas sobre intervenção familiar para portadores de esquizofrenia}

O período coberto pela busca de revisões sistemáticas foi 1980-2006 e os idiomas abrangidos foram inglês, francês, italiano, espanhol e português.

A análise e a seleção das revisões identificadas tiveram como critérios principais: (1) o(s) objetivo(s) da revisão sistemática; (2) a utilização de procedimentos para evitar viés de publicação 17,18; (3) o exame do desenho, da execução e da análise de resultados dos estudos incluídos; (4) o tipo de pacientes incluídos/excluídos; (5) o exame do tipo de intervenção testada (inclusive com base na tipologia de intervenções familiares já referidas) 19,20 e do tipo utilizado como controle dos ensaios incluídos; (6) os desfechos analisados e o exame do instrumental utilizado para a avaliação de desfechos (referido adiante no protocolo da revisão sistemática em pauta).

Nesse sentido, a análise da adequação da metodologia utilizada pelas meta-análises e ensaios foi precedida de análise da evidência sobre fatores prognósticos na condição esquizofrenia e também pelo estudo dos métodos e técnicas utilizadas em procedimentos de intervenção familiar, conforme referido anteriormente.

A identificação de revisões sistemáticas foi feita a partir das bases de estudos Cochrane Library (ano 2001), das publicações relativas ao projeto PORT/Esquizofrenia do governo americano, sobre evidências científicas relativas à atenção a portadores de esquizofrenia, já citadas 4, e da MEDLINE.

A análise preliminar das revisões sistemáticas publicadas na Cochrane Library pelo Schizophrenia Group até o ano de 2002, relativas à intervenção familiar, mostrou que elas abrangeram diagnósticos bastante diferentes; a meta-análise 
selecionada foi Pharoah et al. 15, que focalizou estudos dirigidos apenas a portadores de esquizofrenia e transtornos semelhantes (esquizoafetivos e delirantes). Em nova busca em 2008, foram encontradas versões mais recentes: 2003 e 2006. Analisamos a versão de Pharoah et al. ${ }^{21}$.

Do relatório do estudo PORT/Esquizofrenia, selecionamos inicialmente a revisão de Dixon \& Lehman 13. Da busca realizada no MEDLINE, utilizando as palavras-chave "schizophrenia" AND "family therapy" e os limites english, french, italian, spanish and portuguese, review, meta-analysis, human, encontramos 79 referências, das quais selecionamos 27 resumos, cuja análise resultou na adição de uma revisão sistemática que não constava do resultado das fontes anteriores: o trabalho de Barbato \& D'Avanzo ${ }^{14}$. Numa segunda busca realizada no MEDLINE, utilizando as palavras-chave "schizophrenia" AND "family therapy" e os limites english, french, italian, spanish and portuguese, meta-analysis, human, encontramos quatro referências, das quais duas eram pertinentes: as de Mari \& Streiner 12 e de Pitschel-Walz et al. 16. Os estudos excluídos não eram revisões ou meta-análises, apenas comentários sobre elas.

Após a análise das revisões sistemáticas selecionadas - protocolo, implícito ou explícito, e ensaios por elas incluídos - com base nos critérios acima referidos, concluímos que, embora as meta-análises analisadas apontem evidência de beneficio da intervenção familiar, elas geralmente procederam a exames insuficientes dos processos de cegamento da alocação e da avaliação dos desfechos, bem como das perdas e das formas de análise efetuadas pelos ensaios incluídos, com exceção de Pharoah et al. 21. Além disso, incluíram ensaios que testaram procedimentos não classificáveis em nenhum dos subgrupos teórico-metodológicos focalizados pela presente revisão sistemática, como procedimentos psico-educacionais mal definidos e intervenções familiares dirigidas a grupos de famílias, assim como ensaios de intervenção familiar hospitalar, sem componente comunitário, e procedimentos de intervenção familiar não definidos, conforme detalhado adiante.

\section{Protocolo da presente revisão sistemática}

A meta-análise seguiu protocolo previamente elaborado; as etapas de seleção e de análise de ensaios foram realizadas por duas investigadoras independentes, sendo as divergências resolvidas por discussão e consenso.

\section{- Estratégia de busca de ensaios clínicos}

A estratégia de busca foi baseada no Cochrane Handbook ${ }^{11}$ e no trabalho de Dickersin et al. 22 . Para as pesquisas na MEDLINE e na Cochrane Library foram usadas palavras-chave relacionadas à condição ("schizophrenia", "schizophrenic") e à intervenção ("family therapy”, "family intervention") estudadas. As buscas foram efetuadas no mês de maio de 2007. Além disso, foram realizadas pesquisas em referências bibliográficas das revisões identificadas e analisadas 12,13,14,15,16,21 e dos artigos selecionados. Os idiomas abrangidos foram: inglês, francês, italiano, espanhol e português.

Os ensaios foram selecionados com base nos seguintes critérios: (1) ser um ensaio clínico "placebo" - controlado randomizado de intervenção familiar para a condição esquizofrenia e para transtornos similares (transtornos esquizoafetivos e transtornos delirantes); (2) incluir pacientes em tratamento ambulatorial, compreendendo ou não uma fase inicial hospitalar (durante o surto/recaída); (3) testar uma intervenção familiar que tenha utilizado técnicas comportamentais ou cognitivo-comportamentais ou uma intervenção familiar "pragmática", ou seja, que haja utilizado rudimentos de procedimentos/ técnicas comportamentais, conforme referido na Introdução e Justificativa; (4) analisar o desfecho recaída, nos pacientes, e o desfecho sobrecarga familiar (veja definições adiante).

\section{- Avaliação da pertinência e da qualidade dos ensaios clínicos randomizados}

Procedeu-se ainda a uma avaliação mais apurada da pertinência e a um exame mais detalhado da qualidade dos ensaios, utilizando-se, para esse fim, uma lista de critérios elaborada a partir dos trabalhos de Jüni et al. 23 e de Oxman 20. Ambas as fases de seleção e de análise sistemática da qualidade dos ensaios foram realizadas antes da leitura da seção de resultados dos ensaios, procurando-se evitar a consideração desses resultados naqueles procedimentos.

Os ensaios clínicos "placebo"-controlados selecionados foram avaliados criticamente quanto à sua pertinência levando em conta os objetivos da revisão sistemática (inclusões/exclusões, intervenção e desfechos). O exame da qualidade dos ensaios levou em conta o seu desenho - incluindo os procedimentos de randomização e cegamento, a especificação da intervenção e das co-intervenções, das medidas de desfecho e dos instrumentos de medida -, execução do ensaio e a análise de dados efetuada ou permitida (dados disponíveis). 
A análise da qualidade foi realizada com o objetivo de subsidiar a avaliação da força da evidência produzida por cada um dos ensaios e ratificar ou não a inclusão do estudo na revisão, assim como de subsidiar eventuais análises de sensibilidade relacionadas à qualidade dos ensaios.

Para a realização dessa análise sistemática, mais detalhada, dos ensaios clínicos foi previamente elaborado um protocolo e utilizou-se uma grade correspondente que continha em cada coluna um cabeçalho com as especificações pertinentes (relativas a aspectos do desenho, execução e forma de análise) e linhas correspondentes a cada ensaio selecionado.

Foram avaliadas, entre os aspectos citados: a) Definições/medidas utilizadas para a inclusão/exclusão de pacientes com a explicitação/referência a classificações/definições pertinentes, considerando esquizofrenia ou condições similares, transtorno esquizoafetivo e transtorno delirante, diagnosticados através da Classificação Internacional de Doenças, 10a revisão (CID-10), do Diagnostic and Statistical Manual of Mental Disorders, Fourth Edition (DSM-IV), Diagnostic and Statistical Manual of Mental Disorders, Third Edition (DSM-III), Diagnostic and Statistical Manual of Mental Disorders, Third Edition Revised (DSM-III-R), do Present State Examination (PSE) ou dos Research Diagnostic Criteria (RDC), com exclusão de portadores de condição orgânica.

b) Informações sobre o processo de randomização (processo em si) e tabela pós-randomização para os seguintes fatores prognósticos de recaída: forma de instalação do transtorno, estado civil, nível educacional, problemas na escola (sintomas positivos) e ajustamento social prémórbido (sintomas negativos) 24,25,26,27. Não há evidência de que a cronicidade dos pacientes seja fator prognóstico de recaída. Vários estudos indicam que a emoção expressa (variável que associa medidas de criticismo, hostilidade e intrusão - superenvolvimento) do familiar em relação ao paciente 28,29,30,31,32 é um fator prognóstico de recaída.

c) As etapas do processo de cegamento, nesse caso, cegamento da alocação e do avaliador.

d) As informações disponíveis nos ensaios sobre as intervenções testadas, as intervenções controle (aproximações de placebo) e co-intervenções relevantes. Essas informações foram analisadas para classificar o ensaio em cognitivo-comportamental, comportamental ou "pragmático", para avaliar a validade dos procedimentos realizados para o grupo controle e a intensidade de utilização, por cada grupo comparado, de co-intervenções importantes, ou possivelmente importantes, para os desfechos analisados.

e) Medidas de desfechos utilizadas pelos ensaios.
As definições e medidas de recaída usadas pelos ensaios foram avaliadas tendo como parâmetro a proposta de Brown et al. ${ }^{29}$ : recaída tipo I - recorrência de sintomas em pacientes que estavam sem sintomatologia ao início do estudo, e recaída tipo II - aumento no número e intensidade de sintomas para aqueles pacientes que tinham sintomas esquizofrênicos ativos ao início do estudo. O evento suicídio foi considerado recaída 33 .

O conceito de sobrecarga familiar decorrente da condição esquizofrenia, usado pelos ensaios, foi avaliado tendo como parâmetro a definição de Schene 34 de que as conseqüências para aqueles em contato estreito com um portador de transtorno mental grave, abrangendo as dimensões objetivas, relacionadas ao comportamento do paciente e constituídas pelas tarefas novas e permanentes de cuidado prestado e os problemas intra e extra-familiares associados a cuidar do paciente 35 ; e subjetivas, relacionadas aos sentimentos vividos pelo familiar associados à condição esquizofrenia.

f) As informações disponíveis sobre fluxo de pacientes, incluindo aderência, eventos/intervenções pós-randomização (exclusões, perdas e cross-over) e suas justificativas, e a forma de acompanhamento dos participantes com relação aos desfechos de interesse, foram analisadas para avaliar se a análise de resultados foi efetivamente feita por intenção de tratar e o empenho para promover aderência e manter a integridade dos dados. Os dados disponíveis foram, quando possível, reanalisados por intenção de tratar.

\section{- Análise da heterogeneidade clínica dos ensaios}

Antes de procedermos à análise dos resultados, no sentido de compor uma medida sumária do efeito da intervenção familiar para a condição esquizofrenia, analisamos as fontes de heterogeneidade clínica entre os ensaios, relativas aos fatores prognósticos dos pacientes incluídos nos estudos, às intervenções administradas e às medidas de desfechos utilizadas nos ensaios. A análise da heterogeneidade clínica subsidiou a decisão sobre a validade/utilidade de se estimar uma medida sumária dos resultados. Os testes de heterogeneidade estatística tiveram função complementar à análise da heterogeneidade clínica.

\section{- Análise estatística}

A análise dos dados dos ensaios foi feita utilizando-se o programa estatístico RevMan, versão 4.2.10 (The Cochrane Collaboration, Oxford, Estados Unidos). Foram calculados os riscos relativos (RR) e a diferença de riscos (DR) sumários, 
com os respectivos intervalos de confiança (IC), com nível de significância de 95\%, por meio dos modelos de efeitos fixos (MEF) e de efeitos randômicos (MER). O cálculo da heterogeneidade estatística foi realizado através do teste do $\chi^{2}$, sendo $\mathrm{p}=0,10$ o nível de significância utilizado para considerar homogêneos os resultados.

\section{Resultados}

\section{Resultado da busca}

A partir da referida estratégia de busca, foram capturados, via MEDLINE, 206 artigos, via Cochrane Library, 280 e 11 artigos adicionais via busca em referências bibliográficas. A primeira etapa da seleção pelos títulos excluiu principalmente aqueles que focalizavam tratamentos medicamentosos ou cujo objeto central não era esquizofrenia ou que não se referiam a ensaios clínicos (entre os excluídos, só do MEDLINE, cerca de 28 artigos eram descrições de intervenção familiar ou que tratavam exclusivamente do procedimento "psico-educação para familiares"). Além disso, foram excluídos também sete artigos de periódicos chineses, localizados pela busca em referências bibliográficas (citados por Pharoah et al. 21). Essa primeira etapa resultou em 101 artigos.

Numa análise posterior, ainda preliminar, da pertinência e qualidade dos 101 artigos encontrados sobre intervenção familiar na condição esquizofrenia, selecionamos 11 ensaios clínicos (18 artigos), com base nos critérios acima referidos, a partir dos resumos e dos textos dos ensaios, nessa seqüência. Através da análise dos resumos, foram excluídos 65 artigos que não constituíam ensaios clínicos ou que não testavam uma modalidade nova de intervenção, especificamente elaborada para atender familiares de pacientes. Restaram, então, 36 artigos que tiveram os textos completos avaliados. Dezessete trabalhos foram, finalmente, excluídos: sete, por testarem intervenção indefinida - Vaughan et al. ${ }^{36}$, Schulze Mönking 37, Chien \& Chan 38, Szmukler et al. 39, Merinder et al. ${ }^{40}$, Shimodera et al. 41 e Herz et al. 42 (ensaios que não poderiam ser classificados quanto ao método e técnica dentro dos subgrupos considerados pelo presente estudo, considerando os referenciais acima mencionados, ou com propostas não explicitadas); cinco, porque não constituíam, efetivamente, ensaios clínicos (nesse grupo encontram-se artigos que não possuíam resumo e não foram descartados pelos títulos, ou cuja metodologia não era clara quanto ao desenho de estudo e cujos autores eram também autores de ensaios e por esse mo- tivo foram inicialmente selecionados); dois, por corresponderem a ensaios comparativos 43,44; e dois ensaios, por terem sido realizados exclusivamente em serviço hospitalar 45,46.

$\mathrm{O}$ ensaio publicado em Schooler et al. $47 \mathrm{e}$ Mueser et al. 48 foi excluído por ter procedido a exclusões pós-randomização de cerca de $25 \%$ dos pacientes por critérios de estabilização clínico-medicamentosa, além da perda de mais de $15 \%$ dos pacientes por retirada de consentimento. Os 18 artigos remanescentes correspondem aos 11 ensaios clínicos selecionados (Figura 1).

Os consensos quanto à seleção de artigos foram alcançados depois de discussão entre as duas pesquisadoras que elaboraram a definição da tipologia de intervenções familiares a serem incluídas e as variáveis a serem revisadas sistematicamente e que analisaram independentemente os ensaios 5 , inicialmente a parte metodológica, depois os resultados. Houve divergência inicial quanto à seleção do ensaio de Goldstein et al. 49, o qual pareceu a uma das pesquisadoras ter sido realizado inteiramente em ambiente hospitalar; o consenso foi de que a intervenção familiar havia sido sobretudo ambulatorial. O oposto ocorreu em relação ao ensaio de Glick et al. ${ }^{45}$, excluído, onde houve consenso de que se tratava de ensaio com pacientes internados. Algumas questões, como a dos ensaios quasi-randomizados, foram trabalhadas na análise de sensibilidade. Dúvidas relativas à ocorrência de exclusões pós-randomização e perdas foram analisadas independentemente e consensuadas com a ajuda da terceira autora do presente artigo (vide Colaboradores).

\section{Resultados da análise da pertinência, da qualidade e da heterogeneidade clínica}

Cerca de metade dos ensaios incluíram apenas pacientes com diagnóstico de esquizofrenia 49,50,51,52,53,54,55 o restante incluiu também transtorno esquizoafetivo e transtorno delirante. Alguns ensaios incluíram familiares de pacientes ainda durante a hospitalização destes, outros fizeram a inclusão após a alta; quatro ensaios 56,57,58,59,60 utilizaram critérios de estabilização clínica de pacientes para a inclusão. Além disso, cinco ensaios 50,51,52,53,55,58,61,62 só admitiram familiares com alta emoção expressa em relação ao paciente.

Todos os ensaios informaram terem sido randomizados, exceto os ensaios de Hogarty et al. 56,57 e Tomaras et al. 58 que realizaram processos sistemáticos de seleção, utilizando-se da ordem de chegada ao serviço, sendo classificados como quasi-randomizados. Apesar de cinco ensaios relatarem randomização estratificada, a estratificação não aconteceu com relação a variáveis prog- 


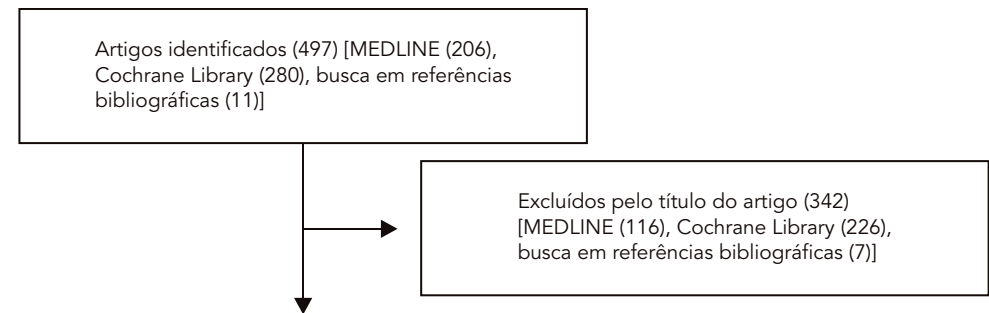

Artigos pré-identificados (148)

União dos subconjuntos (101)

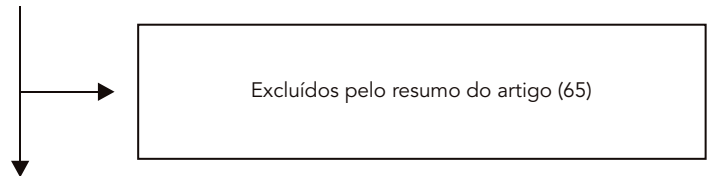

Artigos pré-identificados (36)

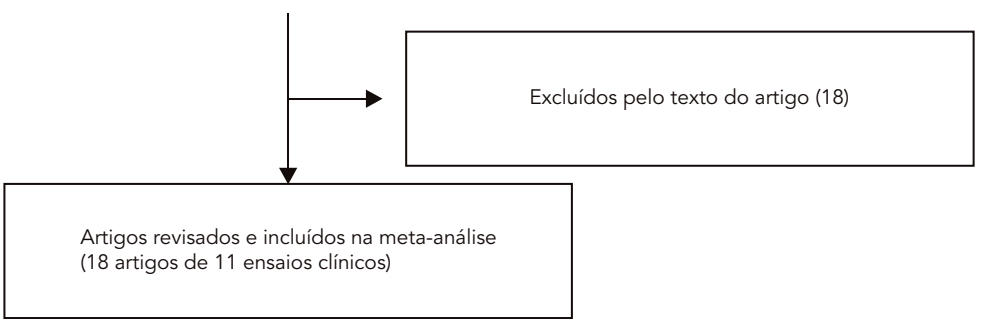

nósticas, anteriormente referidas, identificadas como relevantes pelos estudos disponíveis de boa qualidade. Apenas dois ensaios informaram claramente ter havido cegamento da alocação $59,63,64,65$. Os ensaios, em geral, não incluíram tabela pós-randomização relativa aos fatores prognósticos apontados pela literatura como relevantes para o desfecho recaída, referidos acima.

Em relação à intervenção testada, cinco ensaios foram classificados como de linha pragmática 49,50,51,56,57,58,61,62. Quatro ensaios 52,53,54,59,60,65 foram classificados como comportamentais e dois 55,63,64, como cognitivo-comportamentais.

A duração da intervenção familiar testada pelos ensaios variou de três meses 49 a dois anos 54 sendo em geral inferior a um ano. O trabalho de Rodrigues \& Krauss-Silva 5 descreve os modelos de intervenção familiar investigados e explicita as técnicas aplicadas em cada ensaio da presente revisão sistemática, a partir de referenciais teórico-metodológicos, além de informar características relevantes dos ensaios, como tipo de paciente-família incluído e duração de cada intervenção.

Na maioria dos ensaios selecionados, os pacientes do grupo controle recebeu apenas o cuidado padrão ou usual, do qual constavam vários procedimentos, inclusive medicação. Apenas dois ensaios se preocuparam em elaborar uma intervenção alternativa, aparentemente, com a função placebo. No de Falloon et al. 52 e Falloon \& Pederson 53 o paciente do grupo controle recebeu 25 sessões de psicoterapia individual de suporte durante nove meses. No ensaio de Veltro et al. 60 , os familiares-chave do grupo controle receberam intervenção reabilitadora e de suporte psicológico.

Nos ensaios "pragmáticos", as co-intervenções, além de medicamentos, incluíram sessões individuais de terapia de apoio ou treinamento de habilidades sociais e vocacionais para os pacientes 56,57,58. Nos ensaios comportamentais, 
as co-intervenções também abrangeram o cuidado com a medicação e contemplaram ainda, geralmente, programa de gerenciamento de caso com cuidados ambulatoriais "de rotina", serviço de intervenção em crise, orientação vocacional e aconselhamento para a "reabilitação", para pacientes. Dos dois ensaios classificados como cognitivo-comportamentais, o ensaio de Tarrier et al. 55 mencionou tratamento clínico por uma equipe multiprofissional, o de Barrowclough et al. 63 referiu suporte fornecido por associação de familiares, embora estivesse implícito nos dois ensaios, na avaliação do desfecho recaída, o uso concomitante de medicamentos pelos pacientes.

Com relação aos desfechos, todos os ensaios avaliaram recaída; nenhum ensaio "pragmático" analisou o desfecho sobrecarga familiar. Esse desfecho foi analisado apenas por três ensaios comportamentais, os de Falloon \& Pederson 53, Veltro et al. 60 e Xiong et al. 54 , e por um ensaio cognitivo-comportamental, o de Barrowclough et al. 63 .

Quanto às definições utilizadas pelos ensaios para avaliar recaída, pequenas variações foram observadas, mas em geral as definições foram similares aos citados parâmetros de Brown et al. 29 . Os instrumentos que avaliaram o desfecho recaída foram considerados válidos e similares. Para avaliar a freqüência e duração da recorrência de sintomas e o aumento no número e intensidade de sintomas, foram utilizados, em geral, combinações dos seguintes critérios: (1) modificações na sintomatologia, avaliada periodicamente pelos instrumentos (Brief Psychiatric Rating Scale - BPRS, PSE e outros); (2) mudança no gerenciamento do paciente (aumento na intensidade do monitoramento e/ou mudança na medicação pelo clínico, conforme avaliação feita com base nos prontuários); (3) número e duração de admissões hospitalares, identificadas a partir dos prontuários.

Quatro ensaios utilizaram, para avaliar recaída, o PSE; seis ensaios utilizaram a BPRS; o ensaio de Hogarty et al. 56,57 utilizou o RDC.

Quanto ao cegamento do avaliador, apenas os ensaios comportamentais descritos por Glyn et al. 59 e Randolph et al. 65 , o ensaio de Veltro et al. 60 e os ensaios pragmáticos realizados por Goldstein et al. ${ }^{49}$ e Tomaras et al. 58 informaram a sua ocorrência na avaliação de recaída.

Para o desfecho sobrecarga, os instrumentos e aspectos das dimensões avaliadas diferiram. No ensaio de Falloon \& Pederson 53, o desfecho sobrecarga familiar foi avaliado para o familiarchave, utilizando o instrumento Social Behaviour Assessment Schedule (SBAS) que objetiva fornecer medidas de "interferência na vida familiar atribuída ao paciente" e "sobrecarga subjetiva e desconforto", associados ao distúrbio comportamental do paciente. A avaliação foi realizada no início do estudo e após 9 e 24 meses, de forma cegada.

Xiong et al. 54 informaram que avaliaram a sobrecarga familiar de forma subjetiva utilizando o instrumento de Philips et al. (1990, apud Xiong et al. 54), auto-administrado por um familiar, para avaliar as dimensões: relações intrafamiliares, funcionamento emocional, social e econômico da família, com escalas de 0 a 3 (nenhum a severo).

No ensaio de Barrowclough et al. 63, a sobrecarga familiar foi avaliada de forma cega, no início do estudo e após 6 meses, por escalas de condição de saúde em geral, através do General Health Questionnaire (GHQ; Goldberg \&Willians, 1988, apud Barrowclough et al. 63), por uma avaliação de depressão, através do Beck Depression Inventory (BDI; Beck, 1988, apud Barrowclough et al. 63), e de funcionamento social, medido pela seção D do SBAS (Platt et al. 1980, apud Barrowclough et al. 63). A sobrecarga também foi estimada pela avaliação de "necessidades cardeais", que incluem dimensões de sobrecarga objetiva e subjetiva, seções D e C e E, respectivamente.

No ensaio de Veltro et al. 60 , a sobrecarga foi avaliada através de formulário auto-administrado pelo familiar-chave, adaptado de Morisini et al. (1991, apud Veltro et al. 60), no início do estudo, após um ano, para dimensões objetivas e subjetivas, com quatro níveis de intensidade.

Goldstein et al. ${ }^{49}$ apresentaram cerca de $10 \%$ de perdas e Tomaras et al. 58 mais de $20 \%$ de perdas. Hogarty et al. 56,57 (134 pacientes), outro ensaio "pragmático", teve mais de $20 \%$ de perdas, havendo dez exclusões pós-alocação por não preenchimento dos critérios de inclusão, seis por transferência de hospital e 15 por retirada de consentimento. Além disso, 11 pacientes tiveram recaída no início da intervenção familiar, que não foi concluída, e dois abandonaram o tratamento. Em Leff et al. 50,51, também pragmático, ocorreram cinco exclusões (de 24 pacientes randomizados), por não aderência à medicação: os autores argüiram que o objetivo do estudo foi avaliar o efeito adicional ao medicamento da intervenção familiar e informaram os resultados apenas de três exclusões.

Quanto à forma de análise, três ensaios 49,50,51,52,53 apresentaram os resultados apenas por tratamento efetivo e não foi possível refazêlos por intenção de tratar. O ensaio pragmático de Hogarty et al. 56,57, cujos resultados teriam sido analisados "por intenção de tratar", não incluiu os resultados das perdas relacionadas à retirada de consentimento (15/134) e à transferência (6/134); por esse motivo, refizemos a 
análise por tratamento efetivo. O ensaio cognitivo-comportamental de Tarrier et al. ${ }^{55}$, além de não considerar perdas pós-randomização relativas a consentimento e transferências, incluiu no denominador um número não explicitado de pacientes para os quais os autores não conseguiram estabelecer o desfecho, embora tenham referido análise por intenção de tratar; também, nesse caso, refizemos a análise por tratamento efetivo. Os seis ensaios restantes apresentaram os resultados por intenção de tratar.

\section{Resultados relativos à recaída}

Os testes de heterogeneidade estatística não detectaram heterogeneidade $(\mathrm{p}<0,10)$ entre os ensaios na medida de risco relativo, seja para o conjunto dos ensaios seja para os subgrupos analisados (Figura 2).

Os resultados para recaída aparecem nas Figuras 2 e 3, que mostram medidas sumárias de (RR), pelo MEF, e de DR (Figura 3), pelo modelo de efeitos randômicos, favoráveis à intervenção familiar para o conjunto dos ensaios e para os diferentes subgrupos de intervenção. A medida sumária do RR dos ensaios cognitivo-comportamentais, $[R R=0,43(0,28-0,67)]$, foi equivalente à dos comportamentais $[R R=0,37(0,23-0,60)]$ e à dos "pragmáticos" [(RR $=0,37(0,21-0,66)]$. A DR foi estimada em cerca de $30 \%$, variando de $19 \%$ a $37 \%$, sendo menor no subgrupo dos "pragmáticos", dado que a incidência de recaída nos grupos controle também foi menor nesse subgrupo.

$\mathrm{Na}$ análise de sensibilidade, o resultado favorável à intervenção familiar não foi alterado com a retirada dos dois ensaios pragmáticos em que ocorreu quasi-randomização (em um deles, o de Hogarty et al. 56,57, o volume de perdas foi superior a 20\%) (Tabela 1). Também a retirada dos ensaios que incluíram apenas pacientes com familiares de alta emoção expressa não alterou os resultados.

\section{Resultados relativos à sobrecarga familiar}

Com relação aos resultados para sobrecarga familiar, eles são apresentados para "sobrecarga subjetiva global” em forma gráfica, no ensaio de Falloon \& Pederson 53. Segundo os autores, os resultados revelaram uma redução considerável na sobrecarga global no grupo intervenção familiar, já que somente uma família (de um total de 18) teria apresentado resultado moderado/severo para as medidas de sobrecarga aos 24 meses de estudo. Já no grupo controle, pelo menos metade das famílias apresentaram resultados moderado/severo para as medidas de sobrecarga.
Essa diferença foi estatisticamente significativa $(\mathrm{p}<0,05)$ (Tabela 2).

No ensaio de Xiong et al. ${ }^{54}$, os resultados de sobrecarga familiar foram apresentados como médias da pontuação dos intervalos semestrais (6, 12 e 18 meses) e indicaram diferenças estatisticamente significativas para todas as dimensões avaliadas, exceto para o funcionamento familiar (Tabela 2).

No ensaio de Barrowclough et al. 63, os autores informam que a intervenção familiar teve um pequeno impacto no que compreenderam como sobrecarga familiar, com nenhuma diferença estatisticamente significativa entre os grupos, em relação aos resultados medidos através dos instrumentos GHQ, BDI e SBAS (seção D), que avaliam saúde em geral, depressão e funcionamento social, respectivamente. Quanto ao que denominaram "necessidades cardeais", após 12 meses, houve diferença estatisticamente significativa para os itens D2 (tarefas domésticas) e E1 (emoções negativas associadas com a doença), com melhores resultados no grupo intervenção familiar, não havendo diferença estatisticamente significativa quanto à seção C (relações intrafamiliares), D1 (dificuldades no emprego), D3 (dificuldades financeiras), e D4 (dificuldades sociais) (Tabela 2).

Finalmente, no ensaio de Veltro et al. 60 , as diferenças entre os grupos (intervenção familiar e controle) foram estatisticamente significativas, tanto para sobrecarga objetiva $(\mathrm{p}<0,001)$ quanto para subjetiva ( $\mathrm{p}<0,001)$, após um ano, para as médias da diferença de sobrecarga familiar (Tabela 2).

\section{Discussão}

As revisões sistemáticas já disponíveis sobre a eficácia da intervenção familiar na condição esquizofrenia não preenchem o objetivo do presente estudo e apresentam, em geral, limitações metodológicas importantes, conforme referido anteriormente. A presente revisão sistemática acrescenta a especificação, do ponto de vista de método e técnicas, do procedimento avaliado (intervenção familiar). Essa especificação significa um subsídio mais operacional para o tomador de decisão na eventual incorporação do procedimento intervenção familiar em nossos Centros de Atenção Psicossocial (CAPS).

Para resolver dificuldades iniciais na seleção de ensaios relativas à definição e tipologia de intervenções familiares foi necessário um aprofundamento do conhecimento das autoras sobre teorias, métodos, técnicas e instrumentos no campo cognitivo-comportamental, o que pos- 
Resultados de recaída (risco relativo - RR) para todos os ensaios e subgrupos de ensaios pragmáticos, comportamentais e cognitivo-comportamentais, modelo de efeitos fixos (MEF) de Mantel \& Haenszel.

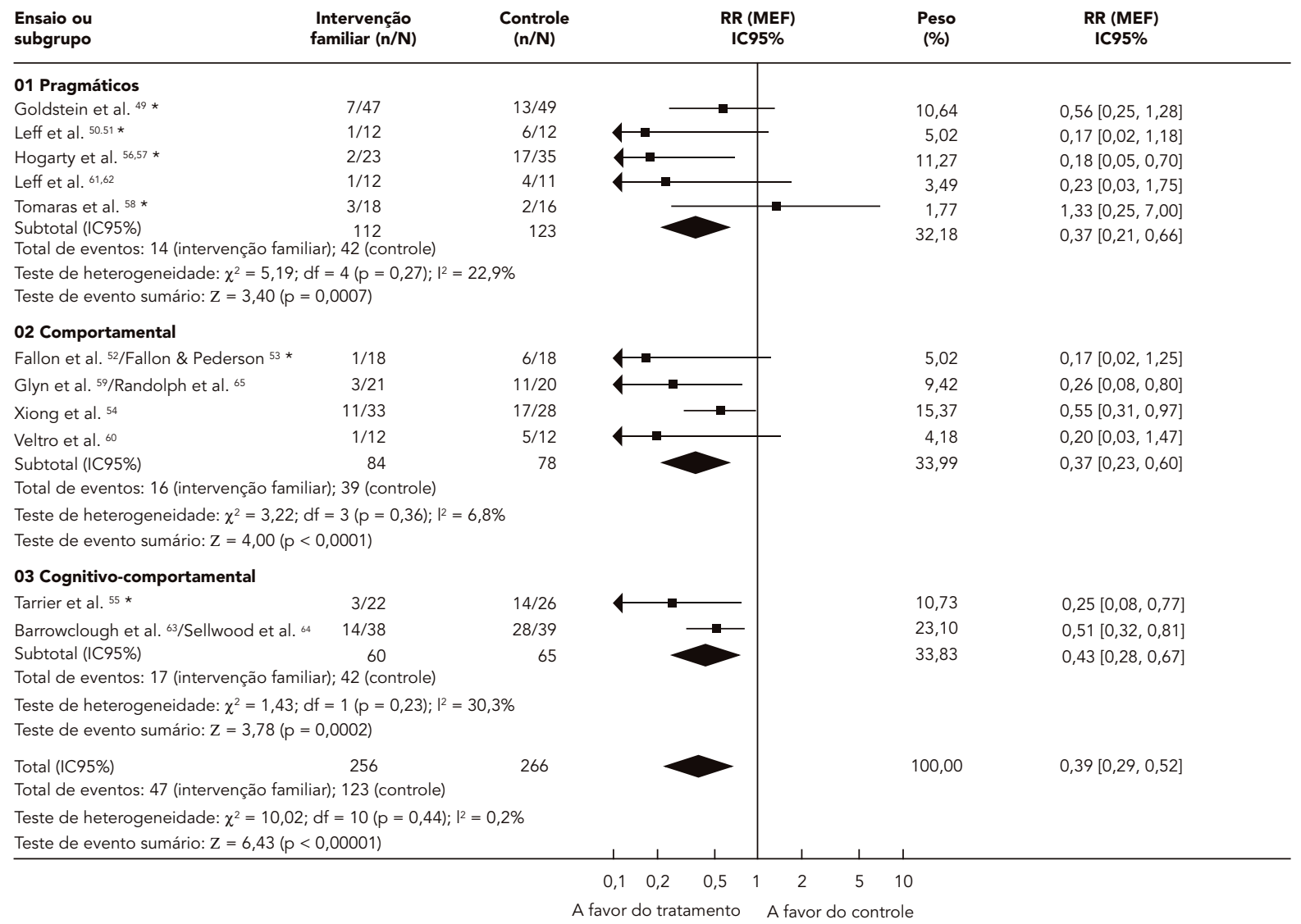

Comparação: 01 intervenção familiar $X$ controle; resultado: 01 recaída.

* Ensaios cuja forma de análise dos dados foi por tratamento efetivo, os demais foram por intenção de tratar.

sibilitou os recortes e classificação referidos na introdução 5 .

As publicações relativas aos ensaios de intervenção familiar, especialmente as da década de 80 , são pouco claras, geralmente, quanto ao desenho, execução e análise do estudo e, freqüentemente, demandaram leituras repetidas para que fossem entendidas. $\mathrm{O}$ artigo de Tarrier et al. 55, por exemplo, compreende dois estudos, que aparecem imbricados no texto. O ensaio de Xiong et al. 54, realizado na China, informa algumas especificidades culturais as quais aparentemente foram importantes na determinação das modificações de técnicas e instrumentos introduzidas no protocolo do ensaio, embora isso não seja explicitado no texto.
Na presente revisão sistemática, foram incluídos ensaios de intervenção familiar cujos grupos controle (aproximações de "placebo") e também co-intervenções diferem entre si, conforme relatado. Assim, as diferenças de eficácia observadas entre os ensaios podem ser, em parte, atribuídas a diferenciais de interação entre os procedimentos dos conjuntos (de procedimentos) comparados.

Os ensaios incluíram percentuais variados de pacientes crônicos; não há evidência, entretanto, de que o grau de cronicidade seja fator prognóstico de recaída. Alguns ensaios incluíram apenas familiares de alta emoção expressa. Os dois ensaios com piores resultados estão nesse grupo, mas diversos fatores podem ter influenciado os resultados. 
Resultados de recaída (diferença de riscos - DR) para todos os ensaios e subgrupos de ensaios pragmáticos, comportamentais e cognitivo-comportamentais, modelo de efeitos randômicos (MER).

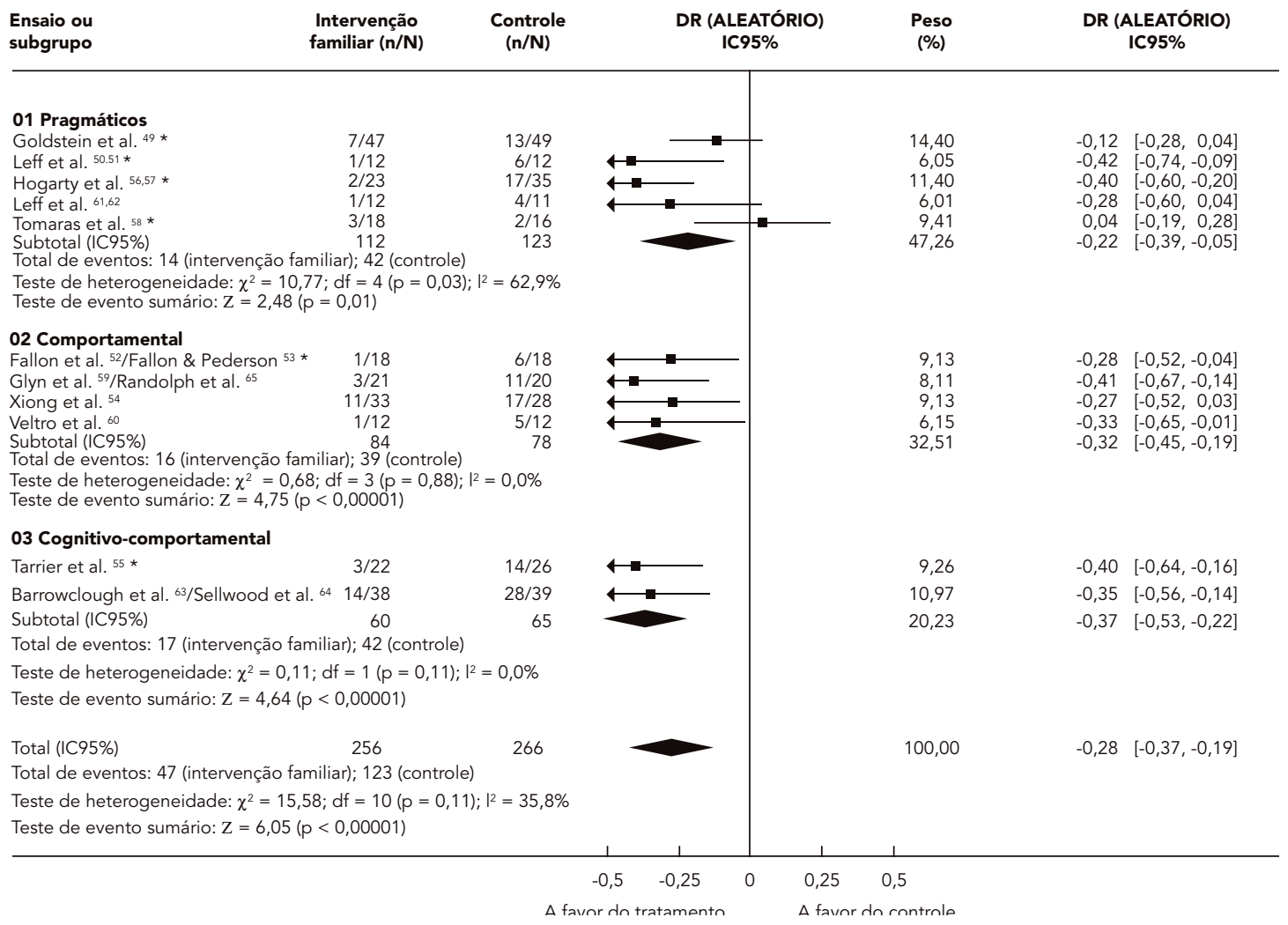

* Ensaios cuja forma de análise dos dados foi por tratamento efetivo, os demais foram por intenção de tratar.

Comparação: 01 intervenção familiar X controle;

Resultado: 01 recaída.

Sobre estabilização clínica dos pacientes e momento de entrada nos ensaios, quatro ensaios 56,57,58,59,60 utilizaram critérios variados/não explicitados de estabilização clínica (três ensaios) ou clínico-medicamentosa (um ensaio) como pré-requisito para inclusão. Embora alguns autores proponham que a intervenção familiar seja iniciada tão cedo quanto possível, inclusive na fase institucional da crise, os resultados daqueles ensaios não indicam que o pré-requisito de estabilização clínica tenha diminuído o percentual observado de eficácia da intervenção familiar.

A análise de sensibilidade para ensaios quasirandomizados 56,57,58 mostrou que eles não influenciaram nos resultados favoráveis à intervenção familiar, para o desfecho recaída, do subgrupo de ensaios "pragmáticos”. Embora esse subgrupo de ensaios tenha apresentado resultados praticamente iguais para recaída que o subgrupo de ensaios comportamentais, e ligeiramente superiores, sem significância estatística, aos do subgrupo de ensaios cognitivo-comportamentais, teoricamente mais elaborados enquanto método de tratamento, a forma de análise dos ensaios "pragmáticos" foi, em geral, por tratamento efetivo, diferentemente da maioria dos ensaios dos outros subgrupos. Houve ainda grandes perdas em ensaios "pragmáticos". A limitação do registro de perdas e falhas na forma de análise de resultados pelos ensaios de intervenção familiar foi também apontada por Falloon 33.

Conforme sugere Falloon 33 , consideramos o evento suicídio, no ensaio de Xiong et al. 54, como recaída, embora esses autores, assim como 
Tabela 1

Resultados para o desfecho recaída das análises de sensibilidade para ensaios quasi-randomizados e para ensaios que incluíram apenas familiares com alta emoção expressa.

\begin{tabular}{lccc}
\hline Grupos de ensaios analisados & Ensaio ( $n$ ) & $\begin{array}{c}\text { Teste de } \\
\text { heterogeneidade }\end{array}$ & $\begin{array}{c}\text { RR (sumário) } \\
\text { MEF }\end{array}$ \\
\hline Todos os ensaios & 11 & $\chi^{2}=10,02 ; p=0,44$ & $0,39(0,29-0,52)$ \\
Retirada dos dois ensaios quasi-randomizados & 9 & $\chi^{2}=6,45 ; p=0,23$ & $0,40(0,30-0,54)$ \\
Retirada dos cinco ensaios que incluíram & 6 & $\chi^{2}=4,66 ; p=0,46$ & $0,43(0,31-0,58)$ \\
apenas famílias com alta emoção expressa & & & \\
\hline
\end{tabular}

RR: risco relativo; MEF: modelo de efeitos fixos.

Tabela 2

Resultados de sobrecarga familiar.

\begin{tabular}{|c|c|c|c|c|c|}
\hline Referência & $\begin{array}{c}\text { Ano de } \\
\text { publicação }\end{array}$ & $\begin{array}{l}\text { Critérios sobrecarga } \\
\text { familiar }\end{array}$ & Instrumentos & $\begin{array}{l}\text { Cegamento } \\
\text { do avaliador }\end{array}$ & Resultados \\
\hline Xiong et al. 54 & 1994 & $\begin{array}{l}\text { Sobrecarga familiar: } \\
\text { intensidade do dano familiar } \\
\text { causado pelo transtorno do } \\
\text { paciente no funcionamento } \\
\text { social, emocional e } \\
\text { econômico da família }\end{array}$ & $\begin{array}{l}\text { Avalia a sobrecarga familiar } \\
\text { de forma subjetiva por meio } \\
\text { do instrumento descrito em } \\
\text { Philips et al. (1990). Avalia } \\
\text { as seguintes dimensões: } \\
\text { relações intrafamiliares, } \\
\text { funcionamento emocional, } \\
\text { social e econômico da } \\
\text { família, com escalas de } 0 \text { a } 3 . \\
\text { (6, } 12 \text { e } 18 \text { meses) }\end{array}$ & $\begin{array}{l}\text { Auto-administrado; não } \\
\text { há informação sobre o } \\
\text { cegamento da avaliação }\end{array}$ & $\begin{array}{l}\text { Os resultados de sobrecarga } \\
\text { familiar são apresentados } \\
\text { como médias da pontuação } \\
\text { dos intervalos semestrais (6, } \\
12 \text { e } 18 \text { meses) e indicam } \\
\text { diferenças estatisticamente } \\
\text { significativas entre os grupos } \\
\text { para todas as dimensões } \\
\text { avaliadas, exceto para o } \\
\text { funcionamento familiar, com } \\
\text { melhores resultados para o } \\
\text { grupo intervenção familiar }\end{array}$ \\
\hline $\begin{array}{l}\text { Falloon et al. } \\
\text { 52/Falloon \& } \\
\text { Pederson } 53\end{array}$ & $1982 / 1985$ & $\begin{array}{c}\text { Ocorrência de sobrecarga e } \\
\text { estresse familiar }\end{array}$ & $\begin{array}{l}\text { Social Behavior Assessment } \\
\text { Scale (SBAS) - familiar mais } \\
\text { envolvido com o papel de } \\
\text { cuidador avaliado no início } \\
\text { do estudo e aos } 9 \text { e } 24 \\
\text { meses. Objetiva fornecer } \\
\text { medidas de "interferência } \\
\text { na vida familiar atribuída ao } \\
\text { paciente" e "sobrecarga } \\
\text { subjetiva e desconforto" }\end{array}$ & Avaliador cegado & $\begin{array}{c}\text { Resultados aos } 24 \text { meses } \\
\text { de estudo: no grupo } \\
\text { intervenção, } 1 \text { família (total } \\
18 \text { famílias) apresentou } \\
\text { resultados moderado/ } \\
\text { severo para as medidas } \\
\text { de sobrecarga; no grupo } \\
\text { controle, pelo menos } \\
\text { metade das famílias (total } \\
18 \text { famílias) apresentaram } \\
\text { resultados moderado/severo. } \\
\text { Diferença estatisticamente } \\
\text { significativa (p<0,05). } \\
\text { Resultados apresentados de } \\
\text { forma gráfica }\end{array}$ \\
\hline
\end{tabular}

(continua) 
Tabela 2 (continuação)

\begin{tabular}{|c|c|c|c|c|c|}
\hline Referência & $\begin{array}{c}\text { Ano de } \\
\text { publicação }\end{array}$ & $\begin{array}{l}\text { Critérios sobrecarga } \\
\text { familiar }\end{array}$ & Instrumentos & $\begin{array}{l}\text { Cegamento } \\
\text { do avaliador }\end{array}$ & Resultados \\
\hline Veltro et al. 60 & 2006 & $\begin{array}{c}\text { Ocorrência de sobrecarga e } \\
\text { estresse familiar }\end{array}$ & $\begin{array}{c}\text { Instrumento adaptado } \\
\text { de Morosini et al. (1991). } \\
\text { Sobrecarga objetiva (12 } \\
\text { itens) sobrecarga subjetiva } \\
\text { (7) hipercriticismo (9); } \\
\text { hiperproteção (8); satisfação } \\
\text { pela intervenção recebida. } \\
\text { Quatro níveis de resposta } \\
\text { de } 1 \text { (problema ausente) a } \\
4 \text { (problema presente em } \\
\text { forma muito grave). Familiar- } \\
\text { chave avaliado no início do } \\
\text { estudo após } 1 \text { e } 11 \text { anos }\end{array}$ & $\begin{array}{l}\text { Auto-administrado; avaliador } \\
\text { cegado } \\
\text { Avaliador cegado }\end{array}$ & $\begin{array}{c}\text { Resultado aos } 12 \text { meses: } \\
\text { diferenças entre os grupos } \\
\text { (médias da diferença } \\
\text { de sobrecarga familiar) } \\
\text { foram estatisticamente } \\
\text { significativas, tanto para } \\
\text { sobrecarga objetiva ( } p< \\
0,001) \text { quanto para subjetiva } \\
(p<0,001)\end{array}$ \\
\hline $\begin{array}{l}\text { Barrowclough } \\
\text { et al. } 63 \text { / } \\
\text { Sellwood } \\
\text { et al. } 64\end{array}$ & 1999/2001 & $\begin{array}{l}\text { Ocorrência de sobrecarga } \\
\text { familiar e mudança no } \\
\text { número de necessidades } \\
\text { cardeais }\end{array}$ & $\begin{array}{c}\text { General Health } \\
\text { Questionnaire (GHQ; } \\
\text { Goldberg \& Williams, 1988), } \\
\text { Beck Depression Inventory } \\
\text { (BDI; Beck, 1988) e o Social } \\
\text { Behavior Assessment } \\
\text { Schedule (seção D somente) } \\
\text { (SBAS; Platt et al., 1980). } \\
\text { A sobrecarga também foi } \\
\text { estimada pela avaliação de } \\
\text { "necessidades cardeais" por } \\
\text { meio da Relatives' Cardinal } \\
\text { Needs Assesment (RCNS; } \\
\text { Barrowclough et al., 1998) }\end{array}$ & & $\begin{array}{c}\text { Resultado aos } 12 \text { meses: } \\
\text { Não houve diferença } \\
\text { estatisticamente significativa } \\
\text { entre os grupos controle } \\
\text { e intervenção familiar, em } \\
\text { relação aos resultados } \\
\text { medidos por meio dos } \\
\text { instrumentos GHQ, } \\
\text { BDI e SBAS (seção D). } \\
\text { Quanto às "necessidades } \\
\text { cardeais" houve diferença } \\
\text { estatisticamente significativa } \\
\text { para os itens D2 (tarefas } \\
\text { domésticas) e E1 (emoções } \\
\text { negativas associadas } \\
\text { com a doença), com } \\
\text { melhores resultados no } \\
\text { grupo intervenção familiar. } \\
\text { Não houve diferença } \\
\text { estatisticamente significativa } \\
\text { quanto à seção C (relações } \\
\text { intrafamiliares), D1 } \\
\text { (dificuldades no emprego), } \\
\text { D3 (dificuldades financeiras), } \\
\text { e D4 (dificuldades sociais) }\end{array}$ \\
\hline
\end{tabular}

Tomaras et al. 58 , o tenham classificado como perda. Não há informação, todavia, sobre suicídio na maioria dos ensaios.

Quanto ao desfecho sobrecarga familiar, a comparação e síntese dos resultados foram limitadas já que eles foram avaliados com instrumentos diferentes e são apresentados de formas diferentes. Schene et al. 66 consideram que alguma padronização dos instrumentos de medida de sobrecarga é necessária e recomendam o desenvolvimento de instrumentos com proprieda- des psicométricas aceitáveis, tanto para pesquisa como para uso na rotina clínica.

Outra limitação da presente revisão sistemática diz respeito à duração dos efeitos das intervenções familiares estudadas. Conforme referido anteriormente, as intervenções familiares testadas pelos ensaios tiveram duração e tempo de acompanhamento dos pacientes para a avaliação dos desfechos recaída e sobrecarga familiar geralmente inferiores a um ano. Apenas o ensaio de Veltro et al. 60 e o de Barrowclough et al. $63 \mathrm{e}$ 
Sellwood et al. 64,67, este com intervenção familiar de seis meses de duração, acompanharam os pacientes por períodos relativamente longos: o primeiro, por 11 anos, para o desfecho hospitalização, e o segundo, por cinco anos, para o desfecho recaída. Quanto à recaída, o resultado foi bastante favorável; todavia, houve, ao longo dos cinco anos de acompanhamento, perda de pouco mais de $20 \%$ dos pacientes, cerca de metade em cada grupo. Além disso, a avaliação de recaída não foi cegada nesse estudo, embora esse desfecho tenha sido cuidadosamente pré-definido. No ensaio de Veltro et al. 60 , o percentual de hospitalização foi semelhante em ambos os grupos, embora perto de $20 \%$ dos pacientes do grupo intervenção e cerca de $50 \%$ do grupo controle tenham sido perdidos, ao cabo dos 11 anos de acompanhamento. O desfecho sobrecarga familiar não foi avaliado no longo prazo por nenhum dos dois ensaios acima.

\section{Resumo}

O objetivo da presente revisão sistemática foi avaliar a eficácia da intervenção familiar de base cognitivocomportamental para portadores de esquizofrenia em tratamento ambulatorial, com relação aos desfechos recaída e sobrecarga familiar. Pesquisadores independentes conduziram as análises da pertinência e da qualidade dos ensaios identificados pela estratégia de busca utilizada, seguindo protocolo previamente elaborado. Foram selecionados 11 ensaios randomizados ou quasi-randomizados. Para o desfecho recaída, o risco relativo sumário, pelo modelo de efeitos fixos, foi favorável à intervenção familiar para o conjunto dos ensaios, com eficácia de cerca de 60\% (50\%-70\%). O risco relativo sumário dos ensaios do subgrupo cognitivo-comportamental $[R R=0,43(0,28-0,67)]$ foi equivalente ao do subgrupo comportamental $[R R=0,37$ $(0,23-0,60)]$ e ao do subgrupo "pragmático" $[R R=0,37$ $(0,21-0,66)]$, embora a forma de análise dos ensaios "pragmáticos" tenha sido, em geral, por tratamento efetivo. A diferença de riscos sumária geral foi estimada em cerca de $30 \%$ pelo modelo de efeitos randômicos. Apenas quatro ensaios analisaram o desfecho sobrecarga familiar, abrangendo, diferentemente, algumas das dimensões objetivas e subjetivas. Os resultados dos estudos individuais foram, em geral, favoráveis à intervenção familiar.

Esquizofrenia; Ensaios Clínicos Aleatórios; Meta-Análise

\section{Conclusão}

A intervenção familiar de base comportamental e cognitivo-comportamental apresenta eficácia de cerca de $60 \%$ (50\%-70\%) para reduzir a probabilidade do desfecho recaída em pacientes esquizofrênicos e esquizoafetivos assistidos ambulatorialmente, o que resulta numa diminuição próxima a $30 \%$ da incidência de recaídas no primeiro ano de tratamento. Não houve diferença estatisticamente significativa entre os subgrupos de ensaios que utilizaram métodos "pragmáticos", comportamentais e cognitivo-comportamentais, embora a forma de análise dos ensaios "pragmáticos" tenha sido, em geral, por tratamento efetivo.

Quanto ao desfecho sobrecarga familiar, os estudos disponíveis, os quais utilizaram diferentes instrumentos, apresentaram resultados, em geral, favoráveis à intervenção familiar, tanto para dimensões objetivas quanto para dimensões subjetivas da sobrecarga.

\section{Colaboradores}

M. G. A. Rodrigues e L. Krauss-Silva participaram na discussão dos métodos, técnicas e instrumentos de intervenção familiar; desenho do protocolo da revisão sistemática e; preparação e edição do manuscrito. M. G. A. Rodrigues e A. C. M. Martins colaboraram na busca bibliográfica. Krauss-Silva e A. C. M. Martins contribuíram na análise estatística. As três autoras participaram da extração, análise, e interpretação de dados.

\section{Agradecimentos}

À Camila Belo, pelo paciente, acurado e rápido tratamento bibliográfico do texto. Às fontes de financiamento: Fundação Carlos Chagas Filho de Amparo à Pesquisa do Estado do Rio de Janeiro (FAPERJ) e Escola Nacional de Saúde Pública Sergio Arouca, da Fundação Oswaldo Cruz. 


\section{Referências}

1. Kapczinski F, Izquierdo I, Quevedo J. Bases Biológicas dos transtornos psiquiátricos. Porto Alegre: Editora Artmed; 2003.

2. Kaplan HI, Sadock BJ. Comprehensive textbook of psychiatry. Baltimore: Willians \& Willians; 1999.

3. Health Systems Research Unit, Clarke Institute of Psychiatry. Review of best practices in mental health reform. Ottawa: Mental Health; 1997.

4. U.S.A. Department of Health and Human Services. Mental health: a report of the surgeon general - executive summary. Rockville: Substance Abuse and Mental Health Services Administration, U.S. Department of Health and Human Services, Center for Mental Health Services, National Institutes of Health, National Institutes of Mental Health; 1999.

5. Rodrigues MGA, Krauss-Silva L. Intervenção familiar na esquizofrenia: recorte de modelos de ensaios clínicos. Rev Dep Psicol UFF 2006; 18:93-109.

6. Bandura A. Social learning theory. New Jersey: Prentice-Hall; 1977.

7. Beck AT. Cognitive therapy and emotional disorders. Boston: International Universities Press; 1979.

8. Caballo VE. O treinamento em habilidades sociais. In: Caballo VE, organizador. Manual de técnicas de terapia e modificação do comportamento. São Paulo: Editora Santos; 1996. p. 361-98.

9. Dobson KS, Dozois DJ. Historical and philosophical bases of the cognitive-behavioral therapies. In: Dobson KS, editor. Handbook of cognitive-behavioral therapies. New York: The Guilford Press; 2001. p. 3-39.

10. Mulrow C. Rationale for systematic reviews. In: Chalmers I, Altman DG, editors. Systematic reviews. 3rd Ed. London: BMJ Publishing Group; 1996. p. 1-8.

11. Higgins JPT, Green S, editors. Cochrane Handbook for systematic reviews of interventions 4.2.6 (updated September 2006). http://www.cochrane. org/resources/handbook/hbook.htm (acessado em 06/Abr/2008).

12. Mari JJ, Streiner D. An overview of family intervention and relapse on schizophrenia: meta-analysis of research findings. Psychol Med 1994; 24:565-78.

13. Dixon LB, Lehman AF. Family interventions for schizophrenia. Schizophr Bull 1995; 21:631-43.

14. Barbato A, D'Avanzo B. Family interventions in schizophrenia and related disorders: a critical review of clinical trials. Acta Psychiatr Scand 2000; 102:81-97.

15. Pharoah FM, Mari JJ, Streiner D. Family intervention for schizophrenia. In: The Cochrane Library, Issue 3, 2001. Oxford: Update Software

16. Pitschel-Walz G, Leucht S, Bäuml J, Kissling W, Engel RR. The effect of family interventions on relapse and rehospitalization in schizophrenia: a meta-analysis. Schizophr Bull 2001; 27:73-92.

17. Chalmers I, Enkin M, Keirse MJ. Preparing and updating systematic reviews of randomized controlled trials of health care. Milbank Q 1993; 71:411-37.

18. Clarke MJ, Stewart LA. Obtaining data from randomised controlled trials: how much do we need for reliable and informative meta-analyses? BMJ 1994; 309:1007-10.
19. Sacks HS, Berrier J, Reitman D, Ancona-Berk VA, Chalmers TC. Meta-analyses of randomized controlled trials. N Engl J Med 1987; 316:450-5.

20. Oxman AD. Checklists for review articles. BMJ 1994; 309:648-51.

21. Pharoah F, Mari J, Rathbone J, Wong W. Family in tervention for schizophrenia (Cochrane Review). In: The Cochrane Library, Issue 4, 2006. Oxford: Update Software.

22. Dickersin K, Scherer R, Lefebvre C. Identifying relevant studies for systematic reviews. BMJ 1994; 309:1286-91.

23. Jüni P, Altman DG, Egger M. Assessing the quality of randomized controlled trials. In: Egger M, Smith GD, Altman DG, editors. Systematic reviews in health care: meta-analysis in context. $2^{\text {nd }}$ Ed. London: BMJ Publishing Group; 2001.

24. Jablensky A, Sartorius N, Ernberg G, Anker M, Korten A, Cooper JE, et al. Schizophrenia: manifestations, incidence and course in different cultures. A World Health Organization ten-country study. Psychol Med Monogr Suppl 1992; 20:1-97.

25. Wieselgren I-M, Lindström LH. A prospective 1-5 year outcome study in first-admitted and readmitted schizophrenic patients; relationship to heredity, premorbid adjustment, duration of disease and education level at index admission and neuroleptic treatment. Acta Psychiatr Scand 1996; 93:9-19.

26. Harrison G, Croudace T, Mason P, Glazebrook C, Medley I. Predicting the long-term outcome of schizophrenia. Psychol Med 1996; 26:697-705.

27. Van Os J, Jones P, Sham P, Bebbington P, Murray RM. Risk factors for onset and persistence of psychosis. Soc Psychiatry Psychiatr Epidemiol 1998; 33:596-605.

28. Brown GW, Monck EM, Castairs GM, Wing JK: Influence of family life on the course of schizophrenic illness. Br J Prev Soc Med 1962; 16:55-68.

29. Brown GW, Birley JLT, Wing JK. Influence of family life on the course of schizophrenic disorders: a replication. Br J Psychiatry 1972; 121:241-58.

30. Vaughan C, Leff JP. The measurement of expressed emotion in the families of psychiatric patients. $\mathrm{Br} \mathrm{J}$ Soc Clin Psychol 1976; 15:157-65.

31. Bebbington P, Kuipers L. The clinical utility of expressed emotion in schizophrenia. Acta Psychiatr Scand Suppl 1994; 382:46-53.

32. Butzlaff RL, Hooley JM. Expressed emotion and psychiatric relapse: a meta-analysis. Arch Gen Psychiatry 1998; 55:547-52.

33. Falloon IRH. Family interventions for mental disorders: efficacy and effectiveness. World Psychiatry $2003 ; 2: 20-8$.

34. Schene AH. Objective and subjective dimensions of family burden. Towards an interactive framework for research. Soc Psychiatry Psychiatr Epidemiol 1990; 25:289-97.

35. Schene AH, Wijngaarden B, Koeter MWJ. Family caregiving in schizophrenia: domains and distress. Schizophr Bull 1998; 24:609-18. 
36. Vaughan K, Doyle M, McConaghy N, Blaszczynski A, Fox A, Tarrier N. The Sydney intervention trial: a controlled trial of relatives' counseling to reduce schizophrenic relapse. Soc Psychiatry Psychiatr Epidemiol 1992; 27:16-21.

37. Schulze Mönking H. Self-help groups for families of schizophrenic patients: formation, development and therapeutic impact. Soc Psychiatry Psychiatr Epidemiol 1994; 29:149-54.

38. Chien WT, Chan SW. One-year follow-up of a multiple-family-group intervention for Chinese families of patients with schizophrenia. Psychiatr Serv 2004; 55:1276-84

39. Szmukler GI, Herrman H, Colusa S, Benson A, Bloch S. A controlled trial of a counselling intervention for caregivers of relatives with schizophrenia. Soc Psychiatry Psychiatr Epidemiol 1996; 31:149-55.

40. Merinder LB, Viuff AG, Laugesen HD, Clemmensen K, Misfelt S, Espensen B. Patient and relative education in community psychiatry: a randomized controlled trial regarding its effectiveness. Soc Psychiatry Psychiatr Epidemiol 1999; 34:287-94.

41. Shimodera S, Inoue S, Mino Y, Tanaka S, Kii M, Motoki Y. Expressed emotion and psychoeducational intervention for relatives of patients with schizophrenia: a randomized controlled study in Japan. Psychiatry Res 2000, 96:141-8.

42. Herz MI, Lamberti JS, Mintz J, Scott R, O’Dell SP, McCartan L, et al. A program for relapse prevention in schizophrenia: a controlled study. Arch Gen Psychiatry 2000; 57:277-83.

43. McFarlane WR, Lukens E, Link B, Dushay R, Deakins SA, Newmark M, et al. Multiple-family groups and psychoeducation in the treatment of schizophrenia. Arch Gen Psychiatry 1995; 52:679-87.

44. Montero I, Asencio A, Hernández I, Masanet MJ, Lacruz M, Bellver F, et al. Two strategies for family intervention in schizophrenia: a randomized trial in a Mediterranean environment. Schizophr Bull 2001; 27:661-70.

45. Glick ID, Clarkin JF, Spencer JH, Haas GL, Lewis AB, Peyser J, et al. A controlled evaluation of inpatient family intervention. Arch Gen Psychiatry 1985; 42:882-6

46. Haas G, Glick ID, Clarking J, Spencer J, Lewis A, Peyser J, et al. Inpatient family intervention: a randomized clinical trial: II Results at hospital discharge. Arch Gen Psychiatry 1988; 45:217-24.

47. Schooler NR, Keith SJ, Severe JB, Matthews SM, Bellack AS, Glick ID, et al. Relapse and rehospitalization during maintenance treatment of schizophrenia. Arch Gen Psychiatry 1997; 54:453-63.

48. Mueser KT, Sengupta A, Schooler NR, Bellack AS, Xie H, Glick ID, et al. Family treatment and medication dosage reduction in schizophrenia: effects on patient social functioning, family attitudes, and burden. J Consult Clin Psychol 2001; 69:3-12.

49. Goldstein MJ, Rodnick EH, Evans JR, May PRA, Steinberg MR. Drug and family therapy in the aftercare of acute schizophrenia. Arch Gen Psychiatry 1978; 35:1169-77.

50. Leff J, Kuipers L, Berkowitz R, Eberlein-Vries, Sturgeon D. A controlled trial of social intervention in the families of schizophrenic patients. Br J Psychiatry 1982; 141:121-34.
51. Leff J, Kuipers L, Berkowitz R, Sturgeon D. A controlled trial of social intervention in the families of schizophrenic patients: two-year follow-up. Br J Psychiatry 1985; 146:594-600.

52. Falloon IR, Boyd JL, McGill CW, Razani J, Moss HB, Gilderman AM. Family management in the prevention of exacerbations of schizophrenia: a controlled study. N Engl J Med 1982; 306:1437-40.

53. Falloon IR, Pederson J. Family management in the prevention of morbidity of schizophrenia: the adjustment of the family unit. Br J Psychiatry 1985; 147:156-63.

54. Xiong W, Philips MR, Hu X, Wang R, Dai Q, Kleinman J, et al. Family-based intervention for schizophrenic patients in China: a randomised controlled trial. Br J Psychiatry 1994; 165:239-47.

55. Tarrier N, Barrowclough C, Vaughn C, Bamrah JS, Porceddu K, Watts S, et al. The community management of schizophrenia: a controlled trial of a behavioural intervention with families to reduce relapse. $\mathrm{Br}$ J Psychiatry 1988; 153:532-42.

56. Hogarty GE, Anderson CM, Reiss DJ, Kornblith SJ, Greenwald DP, Javna CD, et al. Family psychoeducation, social skills training, and maintenance chemotherapy in the aftercare treatment of schizophrenia. Arch Gen Psychiatry 1986; 43:633-42.

57. Hogarty GE, Anderson CM, Reiss DJ, Kornblith SJ, Greenwald DP, Ulrich RF, et al. Family psychoeducation, social skills training, and maintenance chemotherapy in treatment of schizophrenia. Arch Gen Psychiatry 1991; 48:340-7.

58. Tomaras V, Mavreas V, Economou M, Ioannovich E, Karydi V, Stefanis C. The effect of family intervention on chronic schizophrenics under individual psychosocial treatment: a 3-year study. Soc Psychiatry Psychiatr Epidemiol 2000; 35:487-93.

59. Glyn SM, Randolph ET, Eth S, Paz GG, Leong GB, Shaner AL, et al. Schizophrenic symptoms, work adjustment, and behavioral family therapy. Rehabil Psychol 1992; 37:323-39.

60. Veltro F, Magliano L, Morosini P, Fasulo E, Pedicini G, Cascavilla I, Fallon I. Studio controllato randomizzato di un intervento psicoeducativo familiare: esito ad 1 e a 11 anni. Epidemiol Psichiatr Soc 2006; 15:44-51.

61. Leff J, Berkowitz R, Shavit N, Strachan A, Glass I, Vaughn C. A trial of family therapy v. a relatives group for schizophrenia. Br J Psychiatry 1989; 154:58-66.

62. Leff J, Berkowitz R, Shavit N, Strachan A, Glass I, Vaughn C. A trial of family therapy versus a relatives' group for schizophrenia. Two-year follow-up. Br J Psychiatry 1990; 157:571-7.

63. Barrowclough C, Tarrier N, Lewis S, Sellwood W, Mainwaring J, Quinn J, et al. Randomised controlled effectiveness trial of needs-based psychosocial intervention service of carers of people with schizophrenia. Br J Psychiatry 1999; 174:505-11.

64. Sellwood W, Barrowclough C, Tarrier A, Quinn J, Mainwaring J, Lewis S. Needs-based cognitive-behavioral family intervention for carers of patients suffering from schizophrenia: 12-month follow-up. Acta Psychiatr Scand 2001; 104:346-55. 
65. Randolph ET, Eth S, Glynn SM, Paz GG, Leong GB, Shaner AL, et al. Behavioural family management in schizophrenia outcome of a clinic-based intervention. Br J Psychiatry 1994; 164:501-6.

66. Schene AH, Tessler RC, Gamache GM. Instruments measuring family or caregiver burden in severe mental illness. Soc Psychiatry Psychiatr Epidemiol 1994; 29:228-40.
67. Sellwood W, Wittkowski A, Tarrier N, Barrowclough C. Needs-based cognitive-behavioural family intervention for patients suffering from schizophrenia: 5-year follow-up of a randomized controlled effectiveness trial. Acta Psychiatr Scand 2007; 116:447-52.

Recebido em 13/Fev/2008

Versão final reapresentada em 13/Jun/2008 Aprovado em 16/Jun/2008 